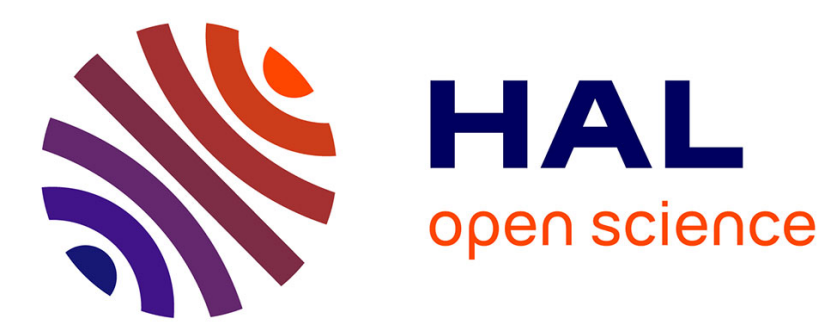

\title{
Experimental demonstration of intermodulation effects in a continuous cesium fountain microwave frequency standard
}

\author{
Jocelyne Guéna, Gregor Dudle, Pierre Thomann
}

\section{To cite this version:}

Jocelyne Guéna, Gregor Dudle, Pierre Thomann. Experimental demonstration of intermodulation effects in a continuous cesium fountain microwave frequency standard. TimeNav'07 EFTF.IEEEFCS'07, May 2007, Genève, Switzerland. 6 p. hal-00165373

\section{HAL Id: hal-00165373 \\ https://hal.science/hal-00165373}

Submitted on 26 Jul 2007

HAL is a multi-disciplinary open access archive for the deposit and dissemination of scientific research documents, whether they are published or not. The documents may come from teaching and research institutions in France or abroad, or from public or private research centers.
L'archive ouverte pluridisciplinaire HAL, est destinée au dépôt et à la diffusion de documents scientifiques de niveau recherche, publiés ou non, émanant des établissements d'enseignement et de recherche français ou étrangers, des laboratoires publics ou privés. 


\section{Experimental demonstration of intermodulation effects in a continuous cesium fountain microwave frequency standard}

\author{
Jocelyne Guéna \\ Present address: LNE-SYRTE \\ 61, Avenue de l'Observatoire \\ F-75014 Paris, France
}

\author{
Gregor Dudle, M. D. Plimmer \\ Federal Office of Metrology \\ Lindenweg 50 \\ CH-3003 Bern-Wabern, Switzerland \\ gregor.dudle@metas.ch
}

\author{
Pierre Thomann \\ LTF-IMT, Université de Neuchâtel \\ Rue A.-L. Breguet 1 \\ CH-2000 Neuchâtel, Switzerland
}

\begin{abstract}
The short-term stability of passive atomic frequency standards, particularly ones operated sequentially, is often limited by local oscillator noise via intermodulation effects. This article describes an experimental demonstration of the intermodulation effect on the frequency stability of a continuous atomic fountain standard usually imperceptible under normal operating conditions. To make the effect observable, we increase the phase instability of the microwave field interrogating the clock transition. We measure the frequency stability of the locked, commercial local oscillator, for both square-wave phase modulation and squarewave frequency modulation of the microwave field. The observed degradation of the stability depends on the modulation frequency in a way that agrees with our earlier theoretical predictions. Most significantly, no degradation is observed when the modulation frequency is made equal to the Ramsey linewidth. When no extra phase noise is added, the frequency instability, currently $\mathbf{2 . 0} \times 10^{-13}$ at $1 \mathrm{~s}$, is limited only by atomic shot-noise. This shows the potential to reduce it via the use of a higher atomic flux.
\end{abstract}

\section{INTRODUCTION}

The ultimate short-term stability of sequentially operated passive frequency standards can be limited by intermodulation effects, often referred to as the Dick effect [1], [2], [3]. The effect was initially invoked in the context of ion trap standards in 1987, and has regained interest with the development of cold cesium fountains [4], most of these devices being operated in a pulsed manner. The intermodulation effect stems from downconversion of local oscillator frequency noise around high harmonics of the cycle rate into the fundamental frequency band of the locking loop, thus leading to a degradation of the short-term stability that can be achieved. In a pulsed fountain it is possible to make the effect negligible if one employs a local oscillator with an exceptionally high stability [5], [6]. An alternative approach would be to guarantee that at all times there are atoms in the region between the two Ramsey pulses by using a juggling or multipulse atomic fountain [7],[8]. Our somewhat more radical strategy is to employ a continuous fountain of laser-cooled atoms.

Earlier theoretical studies, [9], showed that intermodulation effects do occur in a continuous fountain standard but they lie at a much lower level. Contrary to pulsed standards, however, in a continuous fountain these effects can be cancelled completely provided the modulation frequency of the interrogation is chosen appropriately; in short, if this frequency is an odd harmonic of the linewidth of the Ramsey resonance, the intermodulation effect cancels for any modulation scheme. Here, we present an experiment on a continuous fountain in which the above condition is indeed fulfilled and thus demonstrate the suppression of the intermodulation effect. In addition we observe the expected degradation of the short-term stability when it is not.

The remainder of the article is set out as follows. In Section II, we describe the experimental set-up including a brief outline of our continuous fountain, named FOCS-1, and the frequency servo loop. The next section, III, is devoted to the current performance of FOCS-1 in terms of flux, signal-tonoise ratio and frequency stability. In Section IV, we outline the theoretical model developed earlier [9] and predict the value of the intermodulation effect expected in the present experiment. Stability measurements are presented in Section $\mathrm{V}$ and their interpretation discussed.

\section{CONTINUOUS FOUNTAIN CLOCK FREQUENCY STANDARD AND INTERROGATION SCHEME}

\section{A. The fountain FOCS-1}

Fig. 1 shows a sketch of FOCS-1. Cesium atoms from the low-velocity tail of a vapour are captured in a continuous 3D optical molasses (OM). The cold atoms are launched vertically with a longitudinal velocity of $\simeq 3.8 \mathrm{~m} / \mathrm{s}$ using a moving optical molasses (up- and down-going beams at $45^{\circ}$ from the vertical, detuned by $\pm 3.2 \mathrm{MHz}$ ). The beam of cold atoms (isotropic temperature about $60 \mu \mathrm{K}$ ) is transversally collimated by two orthogonal 1D optical molasses, which reduces the transverse temperature to about $7 \mu \mathrm{K}$. The cooling beams are tuned to the cross-over $\mathrm{F}=4 \rightarrow \mathrm{F}^{\prime}=3 / \mathrm{F}=4 \rightarrow \mathrm{F}^{\prime}=5$ of the hyperfine components of the $\mathrm{D} 2$ line, i.e. $25 \mathrm{MHz}$ above the $\mathrm{F}=4 \rightarrow \mathrm{F}^{\prime}=4$ component. A repumper beam tuned to the $6 \mathrm{~S}_{1 / 2}, \mathrm{~F}=3 \rightarrow 6 P_{3 / 2}, \mathrm{~F}^{\prime}=4$ transition is superposed so that all atoms are cooled in the $\mathrm{F}=4$ ground state hyperfine level. 


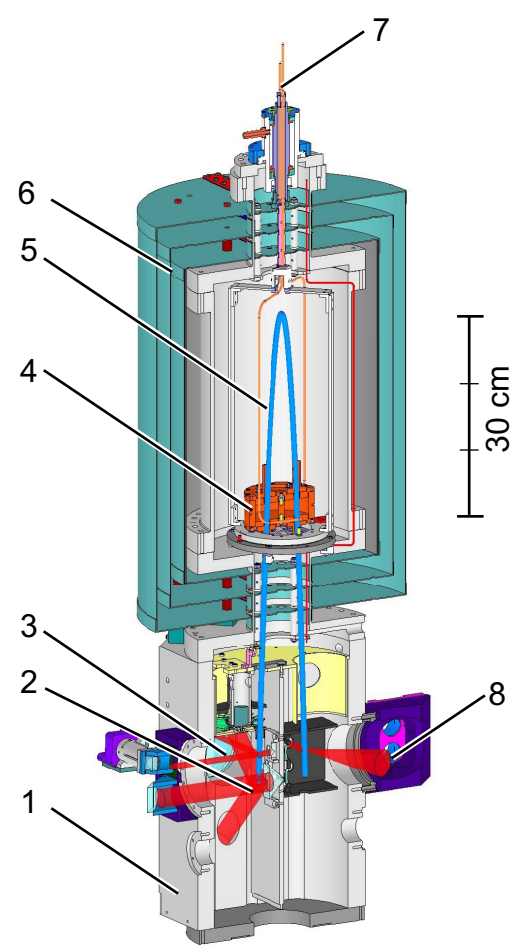

Fig. 1. Cut-away view of the fountain FOCS-1. 1: UHV chamber; 2: optical molasses capture and launch region; 3: transverse collimation; 4: microwave cavity; 5: parabolic flight; 6: mu-metal shielding; 7: microwave field feedthrough; 8: detection beam for atoms in $\mathrm{F}=4$. The apogee of the parabola is about $30 \mathrm{~cm}$ above the microwave cavity. This corresponds to a time between the two Rabi interactions of $0.5 \mathrm{~s}$

In a continuous fountain, the two Rabi interactions must be spatially separated. To achieve this here, the transverse cooling plane is tilted by about $1.8^{\circ}$ from horizontal and the atoms describe an open parabola with a flight time $\mathrm{T} \simeq 0.5 \mathrm{~s}$ in between the two Rabi interaction zones (yielding a Ramsey fringe of $\mathrm{FWHM} \simeq 1 \mathrm{~Hz}$ ). In the present set-up the cold atoms in $\mathrm{F}=4$ are partially pumped into $\mathrm{F}=3$ by the fluorescent light scattered by the OM source. Since this depumping is inevitable, we complete the transfer into $\mathrm{F}=3$ using a transverse laser beam above the cooling plane, tuned to the $\mathrm{F}=4 \rightarrow$ $\mathrm{F}^{\prime}=4$ hyperfine component. The remaining atomic population in $\mathrm{F}=4, \mathrm{~m}_{F} \neq 0$, typically $40 \%$ of the population inversion of the clock transition $\mathrm{F}=3, \mathrm{~m}_{F}=0 \rightarrow \mathrm{F}=4, \mathrm{~m}_{F}=0$, is a source of noise.

The transit time through the microwave cavity is roughly $10 \mathrm{~ms}$. A $\mathrm{C}$-field $\mathrm{B} \approx 70 \mathrm{nT}$ defines the vertical quantisation axis and raises the degeneracy of the $\mathrm{F}=3, \mathrm{~m}_{F} \rightarrow \mathrm{F}=4, \mathrm{~m}_{F}$ microwave transitions. After the second Rabi interaction, the population in $\mathrm{F}=4$ only is detected by induced fluorescence on the $\mathrm{F}=4 \rightarrow \mathrm{F}^{\prime}=5$ optical cycling transition (no repumper laser is used). The detection efficiency limited by the light collection solid angle is a few percent. With a probe laser power $1.5 \mathrm{~mW}$ and waist diameter $10 \mathrm{~mm}$, the number of photons detected per atom is $\gg 1$, so that the detected is limited by atomic shot noise. The photodiode, used with a current-to- voltage

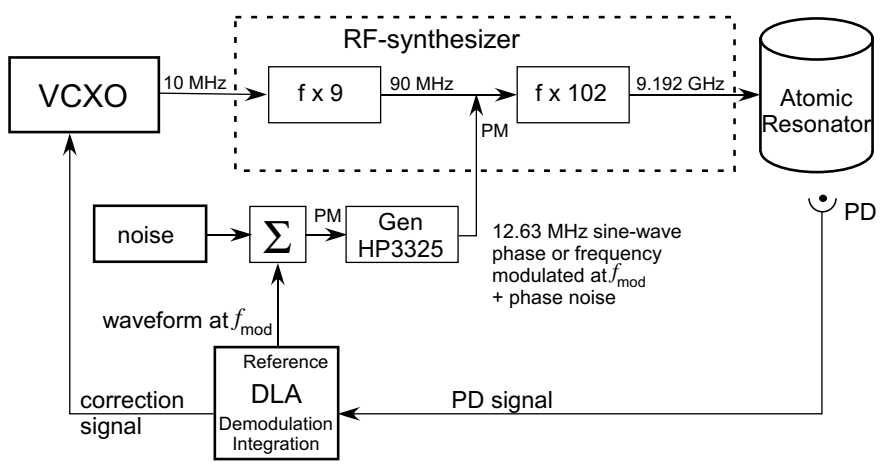

Fig. 2. Layout of the frequency control loop. VCXO: voltage-controlled quartz crystal oscillator; PD: photodiode. DLA: digital lock-in amplifier. Gen: RF synthesiser. PM: phase modulation. The use of the noise generator (noise) and summing amplifier $\Sigma$ is described in Sect. V.

converter with a feedback resistance $1 \mathrm{G} \Omega$, has low dark noise $\left(4 \times 10^{-15} \mathrm{~A} \mathrm{~Hz}^{-1 / 2}\right)$. The voltage output is further amplified by a factor of 100 .

\section{B. Continuous Ramsey microwave interrogation}

The layout of the frequency servo loop is illustrated in Fig. 2. We begin with a commercial voltage-controlled quartz crystal oscillator VCXO (Oscilloquartz BVA 8607) displaying an Allan deviation of $10^{-13}$ up to $100 \mathrm{~s}$. A frequency synthesiser multiplies the $10 \mathrm{MHz}$ output of the VCXO to $9180 \mathrm{MHz}$ and applies a phase modulation at $12.6 \mathrm{MHz}$ provided by an external function generator (HP3325 synthesiser). In this way we generate a tunable microwave field with a carrier frequency at the clock transition frequency (9 $192631770 \mathrm{~Hz}$ ) and an amplitude adjusted to induce $\pi / 2$ Rabi pulses in the Ramsey cavity. To generate the error signal for frequency correction, the phase of the $12.6 \mathrm{MHz}$ oscillation from the synthesiser is square-wave modulated with a p.p. amplitude of $\pi / 2$. The phase of the microwave field at the clock frequency is thus modulated with an identical amplitude and waveform. The waveform, frequency $f_{\text {mod }}$ and amplitude of the phase modulation are controlled by the reference output of a digital lockin amplifier (DLA). After square-wave demodulation of the fluorescence photodetector signal in the DLA, the demodulator output is integrated and the correction voltage applied to the VCXO. The locking loop is controlled using LabView RT in a PXI-system.

\section{SIGNAL-TO-NOISE RATIO AND CURRENT FREQUENCY STABILITY}

In a continuous fountain, if all sources of technical noise are suppressed, the achievable stability is limited only by the signal-to-noise ratio $S / N$ of the detected atomic flux which itself scales with the square root of this flux (atomic shot noise limit). The Allan deviation $\sigma_{y}$, a measure of the frequency instability of the fountain, is then expected to fall as the inverse square root of the atomic flux, $\sigma_{y} \propto \phi^{-1 / 2}$. To check that our fountain satisfies these conditions, we first studied how the noise varied when the atomic flux was modified either i) by a 
change in the power of the repumper laser or ii) by modifying the power of the cooling beams of the 3D-optical molasses.
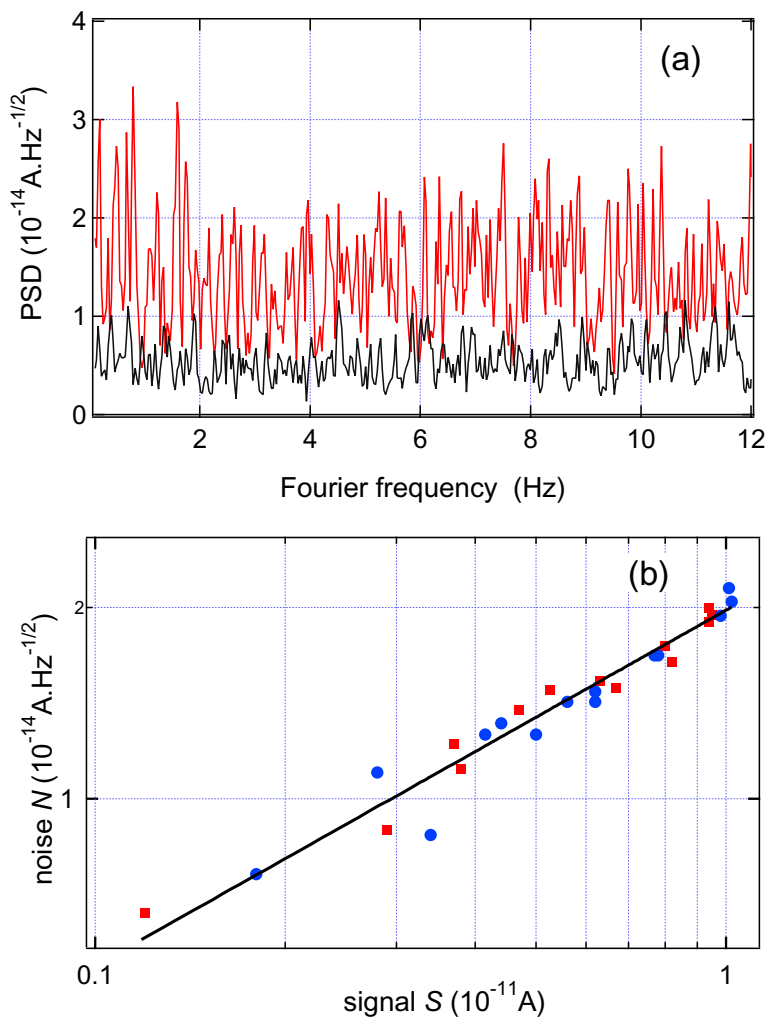

Fig. 3. Rms values of the noise $N$ of the fluorescence photodetector signal. (a) $v s$ the Fourier frequency at intermediate atomic flux (upper trace) and with no atomic flux (lower trace). (b) $v s$ the contribution $S$ of the atomic fluorescence to the DC signal. The microwave frequency is tuned to the centre of the central Ramsey fringe of the clock transition without phase modulation. We modify the atomic flux by varying the power of the repumper beam ( $\square$ ) or of the cooling beams $(\bullet)$ in the moving optical molasses source. The solid line is a fit to a power law with exponent $0.560 \pm 0.025$.

The noise of the fluorescence photodetector signal, measured using a spectrum analyser, was observed to be white between $0.1 \mathrm{~Hz}$ and $20 \mathrm{~Hz}$ and dominated by the atomic fluorescence contribution, as exemplified in Fig. 3a. Figure $3 b$ displays the measured noise as a function of the detected atomic flux for both methods. The small noise contribution from the background signal due to scattered probe light $\left(\sim 5 \times 10^{-15} \mathrm{~A} \mathrm{~Hz}^{-1 / 2}\right)$ has been subtracted quadratically. The experimental data are fitted with a power law $N=S^{k}$ where $N$ represents the noise and $S$ the atomic signal. The value obtained for $k$ lies $\sim 2.5 \sigma$ above the value 0.5 expected for pure atomic shot noise. The signal-to-noise ratio can thus provide a measure of the flux of detected atoms, the two being related via

$$
\phi=2(S / N)^{2} .
$$

Using this equation, we infer that for the data point corresponding to the strongest atomic signal, the useful flux ${ }^{1}$ is

\footnotetext{
${ }^{1}$ i.e. the flux of atoms having undergone the clock transition excluding the background atoms in the magnetically sensitive $\mathrm{F}=4, \mathrm{~m}_{F} \neq 0$ sublevels.
}

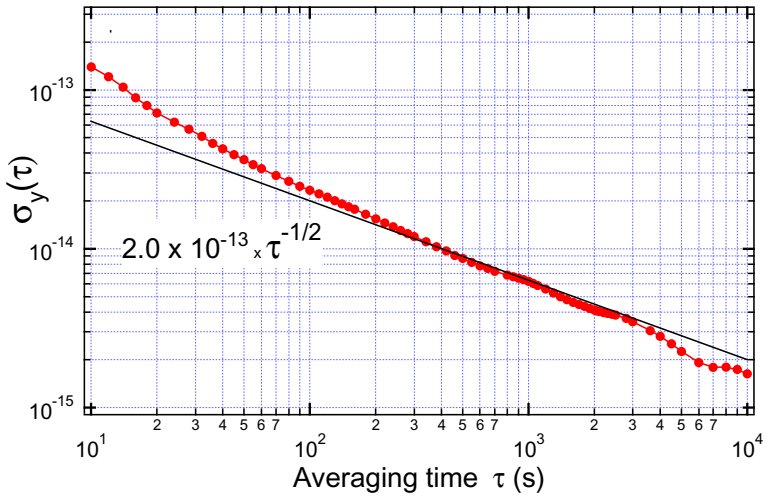

Fig. 4. Allan deviation $\sigma_{y}$ of the frequency difference between the LO locked to FOCS-1 and a reference maser, as a function of the averaging time $\tau$. The points represent the experimental data and the straight line corresponds to $2.0 \times 10^{-13} \tau^{-1 / 2}$.

approximately $2 \times 10^{5}$ at/s.

For frequency stability measurements, the $10 \mathrm{MHz}$ output of the VCXO locked to the fountain is compared with that of a hydrogen maser using a frequency comparator (Vremya $\mathrm{VCH}-314)$. The maser in question (BIPM reference 140-5701) contributes to the maintenance of TAI and $\operatorname{UTC}(\mathrm{CH})$ ([11]) and is thus well characterized. Fig. 4 displays a result obtained at maximum flux. At $1 \mathrm{~s}$, the phase noise of the maser dominates. For $\tau>200 \mathrm{~s}$, the Allan deviation is consistent with $2 \times 10^{-13} \tau^{-1 / 2}$ and lies near the limit $\sim$ (1.5 to 1.8$) \times 10^{-13} \tau^{-1 / 2}$ originating from atomic shot noise expected from the above discussion of the signal-to-noise ratio. In addition, control measurements performed at halfmaximum flux gave results compatible with a degradation of the instability as $\phi^{-1 / 2}$, i.e. directly related to atomic shot noise.

\section{PREDICTED SIZE OF THE INTERMODULATION EFFECT}

\section{A. Allan deviation arising from the intermodulation effect}

A detailed theoretical analysis of a possible increase in the instability of a frequency standard due to intermodulation effects has already been published [9]. Here we recall only the essential ingredients of the model and the main results derived. From a knowledge of the power spectral density (PSD) of the frequency fluctuations of the free running oscillator $S_{y}^{L O}(f)$, the aim of the model is to predict the power spectral density $S_{y}^{L L O}(f)$ of the oscillator locked to a Ramsey resonator using any modulation-demodulation scheme. Once the PSD of the locked oscillator is known, the Allan deviation can then be calculated. The spectrum of the LLO consists of two components: the first corresponds to the error signal that controls the LO frequency fluctuations; the second, not reduced by the loop gain, contains all even harmonics of the modulation frequency $f_{\text {mod }}$ and corresponds to a spurious signal generated by down-conversion of the LO frequency fluctuations at harmonics of the modulation frequency. The latter is the aliasing part of interest here and is given by [9]: 


$$
S_{y, D c}^{L L O}(f) \simeq 2 \sum_{k=1}^{\infty} \frac{\left|c_{2 k}\right|^{2}}{c_{0}^{2}} \operatorname{sinc}^{2}\left(2 k \pi f_{\text {mod }} T\right) S_{y}^{L O}\left(2 k f_{\text {mod }}\right)
$$

for Fourier frequencies $0 \leq f \leq f_{F}$, where $\operatorname{sinc}(\mathrm{x})=$ $\sin (\mathrm{x}) / \mathrm{x}$, the $c_{2 k}$ are the Fourier coefficients of the modulationdemodulation function, $T$ is an effective transit time between the two Ramsey pulses (here $\simeq 0.49 \mathrm{~s}$ ) and $f_{F}$ is the bandwidth of the low-pass filter in the loop. This equation has the remarkable property that for $T=T_{\bmod } / 2$, i.e. when the modulation frequency is equal to the resonator linewidth $\Delta \nu_{0}(\simeq 1 \mathrm{~Hz})$, all terms in Eq.(1) vanish and with them the aliasing effect, whatever the modulation-demodulation scheme employed. This condition can be interpreted as a filtering effect of the LO frequency fluctuations at the even harmonics of $f_{\text {mod }}$ by averaging over the transit time through the resonator. Furthermore, in the particular case of square-wave phase modulation and square-wave demodulation, the coefficients $c_{2 k}$ vanish for all values of $k$, which provides even more robust cancellation of intermodulation noise.

Within the bandwidth of the frequency control loop $f_{F}$ (a fraction of a hertz), the frequency perturbations $S_{y, D c}^{L L O}(f)$ due to the continuous intermodulation effect can be regarded as white noise (Eq. (2)). As a consequence, the Allan variance associated with this effect is given by:

$$
\sigma_{y, D c}^{2}(\tau)=S_{y, D c}^{L L O}(f=0) / 2 \tau
$$

provided the averaging time $\tau$ is longer than the time constant of the servo loop (typically $\tau>10 \mathrm{~s}$ ).

For quantitative predictions, we need to calculate the Fourier coefficients $c_{2 k}$ involved in Eq. 2 and use a model for $S_{y}^{L O}(f)$, the PSD of the frequency fluctuations of the free LO. For $S_{y}^{L O}(f)$, the principal sources of frequency instability can be parameterized by the following expansion:

$$
S_{y}(f)=\sum_{\alpha=-2}^{2} h_{\alpha} \times f^{\alpha}
$$

corresponding to random frequency $\left(h_{-2}\right)$, flicker frequency $\left(h_{-1}\right)$, white frequency $\left(h_{0}\right)$, flicker phase $\left(h_{1}\right)$ and white phase noise $\left(h_{2}\right)$.

In Ref. [10], three usual types of modulation schemes were dealt with: square-wave phase modulation, sine-wave and square-wave frequency modulations. For each one, either first harmonic or wideband demodulation schemes were considered. The simulations of the intermodulation effect were performed for a particular quartz oscillator exhibiting contributions from $h_{-1}$ and $h_{1}$ types of noise, with a flicker floor Allan deviation of $3 \times 10^{-13}$. The results showed that for significant deviation $\left(0.4\right.$ to $0.5 \mathrm{~Hz}$ ) of $f_{\text {mod }}$ from the optimal value $\Delta \nu_{0}=1 \mathrm{~Hz}$ (or its odd harmonics) the Allan deviation due to the intermodulation effect reaches at most a value of $10^{-13}$ for $\tau=1$ s, i.e. one lying below the present Allan deviation limited by the atomic shot noise (see Sec. III where $f_{\text {mod }}$ is chosen equal to $\Delta \nu_{0}=1 \mathrm{~Hz}$ ). Similar predictions were made for each of the three modulation schemes. Under normal working conditions, our present local oscillator has a $\sim 3$ times lower flicker phase noise than that used in the theoretical model. Thus it is not well suited for demonstrating the intermodulation effect. For this reason its phase noise performance was purposely degraded. To do this, we injected white phase noise $\left(h_{2}\right.$-type) with an amplitude such that the intermodulation effect contribution was about twice the atomic shot noise for an experimentally sensible deviation of $f_{\text {mod }}$ from $1 \mathrm{~Hz}$ (or its odd harmonics).

\section{B. Prediction for square-wave phase modulation with white} phase noise

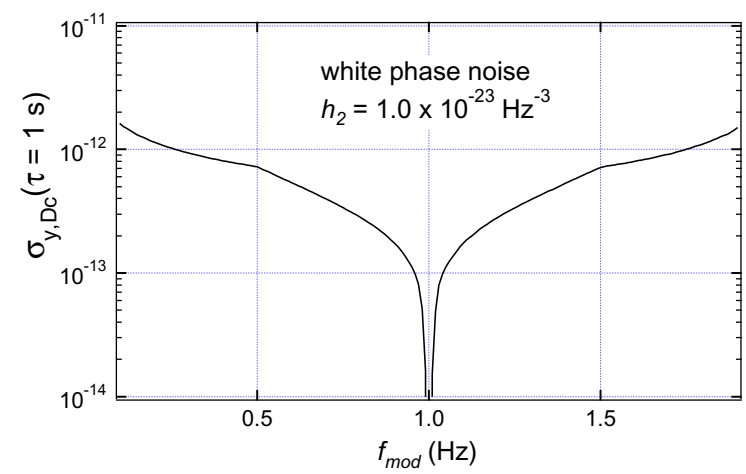

Fig. 5. Allan deviation due to the intermodulation effect using the model developed by Joyet et al. [9] for the case of square-wave phase modulation with white phase noise $h_{2}=10^{-23} \mathrm{~Hz}^{-3}$, as a function of the frequency modulation $f_{\text {mod }}$. Values calculated for an averaging time $\tau=1 \mathrm{~s}$.

For square-wave phase modulation and wideband demodulation, the Fourier coefficients can be calculated analytically and the $h_{2}$ contribution to the intermodulation effect is given by [10]:

$$
\sigma_{y, D c}(\xi, \tau)=\frac{1}{2 \pi^{2} \xi(1-|\xi-1|)}\left\{\frac{h_{2}}{T^{2}} \xi^{2} g(\xi)\right\}^{1 / 2} \tau^{-1 / 2}
$$

where $g(\xi)=\sum_{k=0}^{\infty} \sin ^{4}(k \pi \xi) / k^{2}$ and $\xi=f_{\text {mod }} / \Delta \nu_{0}$. The dependence of the Allan deviation on the frequency modulation $f_{\text {mod }}$ is indicated in Fig. 5 for $h_{2}=10^{-23} \mathrm{~Hz}^{-3}$. From the relation $S_{\phi}(f)=S_{y}(f) \times\left(f_{0} / f\right)^{2}$ with $f_{0} \simeq 9.2 \mathrm{GHz}$ the clock frequency, this corresponds to a PSD of the phase noise $S_{\phi}(f)$ of $10^{-3} \mathrm{rad}^{2} \mathrm{~Hz}^{-1}$. The obvious feature is the notch effect of the continuous Ramsey resonator for the ideal modulation frequency of $1 \mathrm{~Hz}$, i.e. equal to the width $\Delta \nu_{0}$ of the resonance line, and the rapid rise in instability either side of this value.

\section{NOISE GENERATION, MEASUREMENTS AND RESUlTS}

To increase the (usually small) phase noise of the microwave field, we add a noise voltage to the phase modulation input of the $12.6 \mathrm{MHz}$ function generator (see Fig. 2). The noise voltage provided by a wideband white noise generator is lowpass filtered and amplified beforehand. We adjust the cut-off frequency to select the first 20 harmonics of the modulation 
frequency $f_{\text {mod }}$, since these contribute $>98 \%$ of the intermodulation effect. The rms noise voltage is adjusted to provide a phase noise of about $\sim 30 \mathrm{mrad} \mathrm{Hz}{ }^{-1 / 2}\left(h_{2} \approx 10^{-23} \mathrm{~Hz}^{-3}\right)$. Note that because we inject the phase noise into the $12.6 \mathrm{MHz}$ oscillation, rather than directly into the VCXO control voltage input, the added frequency instability of the VCXO is then representative of the intermodulation effect, and not of some parasitic effect due to a direct degradation of the VCXO.
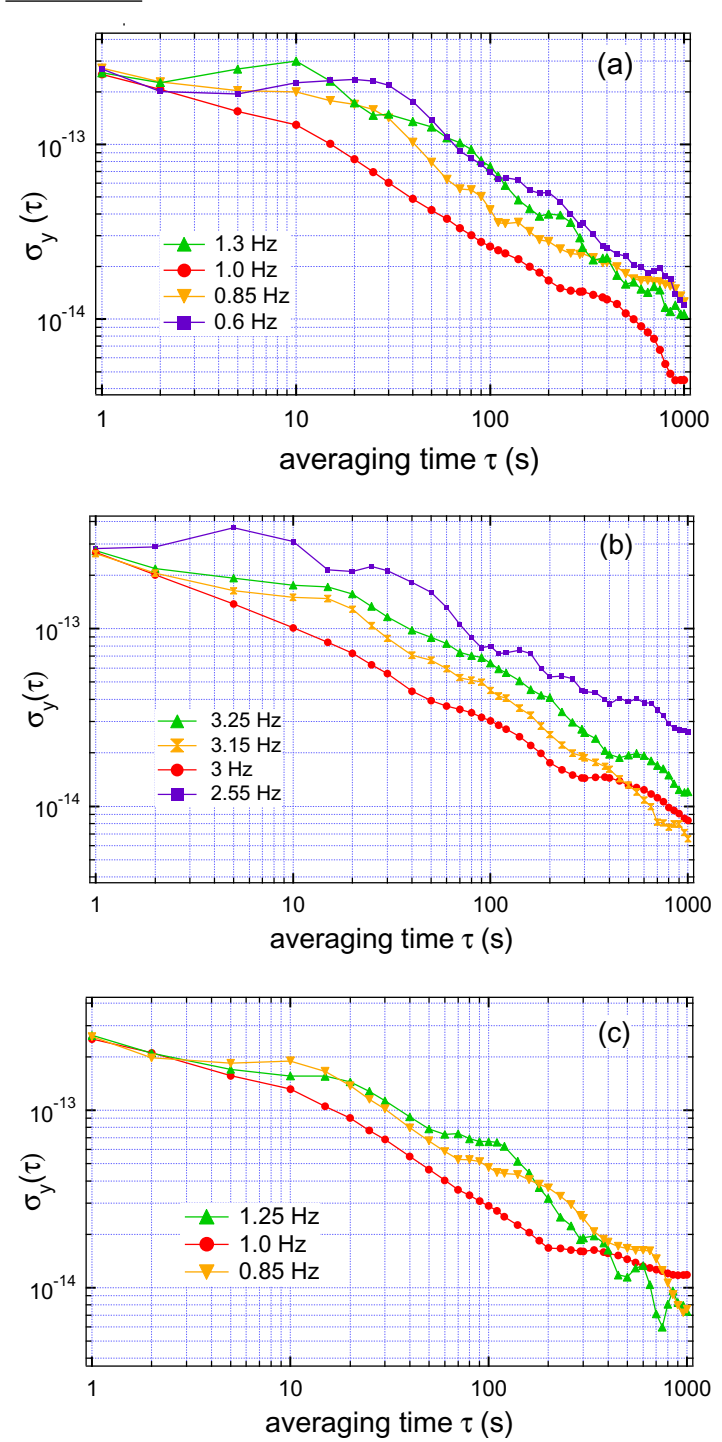

Fig. 6. Examples of experimental Allan deviations of the LO locked to the continuous fountain standard FOCS-1 with white phase noise injected via the synthesiser into the microwave field fed to the Ramsey resonator. (a) Squarewave phase modulation with modulation frequency varied around $1 \mathrm{~Hz}$, the (FWHM) linewidth of the resonator. (b) Square-wave phase modulation with modulation frequency varied around $3 \mathrm{~Hz}$. (c) Square-wave frequency modulation with modulation frequency varied around $1 \mathrm{~Hz}$.

Most of the frequency instability data were acquired using square-wave phase modulation with $f_{\text {mod }}$ varied around either $1 \mathrm{~Hz}$ or $3 \mathrm{~Hz}$, i.e. the first or third harmonic of the Ramsey resonator linewidth. For each value of $f_{\text {mod }}$, the Allan deviations, obtained after a measurement time of typically $7000 \mathrm{~s}$ are plotted in Figs. 6a and 6b, respectively. For a time $\tau$ of a few hundred seconds, the VCXO is locked to the fountain and the Allan deviations approach the $\tau^{-1 / 2}$ law (white frequency noise) expected from both atomic shot noise and the intermodulation effect. For $\tau>1000 \mathrm{~s}$, the statistics are poorer and the values less significant. We should note that, when one alters the modulation frequency, the slope of the Ramsey discriminator is modified. For example when $f_{\text {mod }}=1.4 \mathrm{~Hz}$ (instead of $1.0 \mathrm{~Hz}$ ), the slope is decreased by $30 \%$ in square-wave phase modulation mode ${ }^{2}$. This means that even in the absence of the intermodulation effect, the instability limited by the atomic shot noise can be increased when $f_{\text {mod }}$ is changed. We have allowed for this effect by repeating the measurements without phase noise injected in the synthesiser. The observed degradation of stability due to the change of the discriminator slope agrees with that expected based on atomic shot noise and remains much smaller than the frequency instability observed when phase noise is injected (see below). We also carried out measurements using squarewave frequency modulation. Simulations performed in Ref. [10] indicate that the intermodulation effect should be present with the same order of magnitude. With this modulation scheme, however, the slope is smaller, and is less sensitive to $f_{\text {mod }}$. The corresponding experimental results are plotted in Fig. 6c. In the three graphs of Fig. 6, the better stability obtained for $f_{\bmod }$ equal to $1 \mathrm{~Hz}$ (in (a) and (c)) or $3 \mathrm{~Hz}$ (in (b)) is quite conspicuous.

In order to extract the intermodulation contribution to the measured instabilities and compare them with the prediction above, we subtracted quadratically the instabilities measured without injected phase noise from the total instabilities measured with (added) phase noise present. The values are taken for averaging times $\tau$ in the range 100 to $400 \mathrm{~s}$ and scaled to $1 \mathrm{~s}$ using the $\tau^{-1 / 2}$ law. The results are plotted as a function of $f_{\text {mod }}$ in Fig. 7 and show good agreement with the intermodulation effect expected from theory. The most striking feature is the suppression of the effect when the modulation frequency is set equal to an odd harmonic of the Ramsey fringe linewidth. Note that the model we used to predict the intermodulation effect [9] makes several simplifications. These are that the atomic beam is monokinetic (zero longitudinal temperature), the Rabi pulses infinitely short, hence absence of transients and that there is no phase shift between modulation and demodulation waveforms. One might wonder whether the use of such approximations could lead to an underestimate of the effect. In fact, several points involved in the real experiment were considered in Ref. [10]:

(i) longitudinal velocity distribution of atoms (a few $\mathrm{cm} / \mathrm{s}$ around the average launch velocity of $4 \mathrm{~m} / \mathrm{s}$, or longitudinal temperature of about $75 \mu \mathrm{K}$ );

(ii) phase shift between modulation and demodulation;

\footnotetext{
${ }^{2}$ The phase of the demodulation has also to be readjusted, which we do by maximizing the slope of the Ramsey fringe when the microwave frequency is detuned by half a linewidth $( \pm 0.5 \mathrm{~Hz})$ from its centre.
} 
(iii) introduction of dead times (blanking) of duration equal to the duration of the transients.

The results of the more realistic simulations imply that the corresponding degradations of stability are still expected to be very small compared with the atomic shot noise. Thus we believe that the slight excess of instability we observed over the predicted value arose from some technical noise added in the generation of the phase noise. We recall that the latter corresponds to an increase by two to three orders of magnitude with respect to the undegraded phase noise, and to an $\mathrm{rms}$ phase noise of $2 \%$ of the $\pi / 2$ p.p. phase modulation. An effect of transients might just have shown up when $f_{\text {mod }}$ was varied around $3 \mathrm{~Hz}$ instead of $1 \mathrm{~Hz}$ but no significant increase was observed (see Fig. 7).

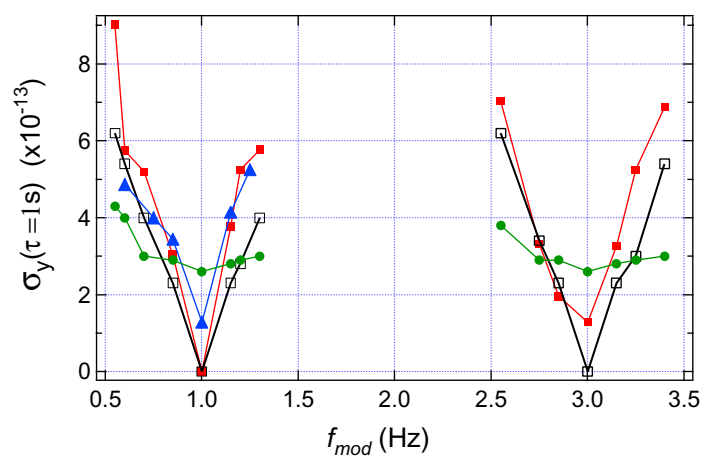

Fig. 7. Allan deviations vs interrogation modulation frequency: $(\bullet)$ observed in square-wave phase modulation without injected phase noise, used as references ; observed with injected phase noise after quadratic subtraction of the references ( $\boldsymbol{\square}$ and $\boldsymbol{\Delta}$ for square-wave phase and square-wave frequency modulations, respectively); predicted intermodulation effect for injected noise $h_{2}=10^{-23} \mathrm{~Hz}^{-3}$ and square-wave modulation ( $\square$ ).

\section{CONCLUSION}

We have shown that a continuous cold-atom fountain clock using a commercial quartz crystal oscillator displays a frequency stability limited mainly by atomic shot noise. By purposely degrading the noise spectrum of the microwave field fed to the Ramsey cavity, we have confirmed the predictions of a model specifically developed to treat the intermodulation effect in a continuous interrogation scheme, in particular the cancellation of this effect when the modulation frequency involved in the microwave frequency locking loop is equals to an odd harmonic of the resonator linewidth (FWHM about $1 \mathrm{~Hz}$ ). When the modulation frequency is changed from these values, the size of the intermodulation effect observed is at the level predicted by the model. This experimental verification is significant since several factors involved in the actual experiment, e.g. transients effects, were difficult to take into account in the model. To observe the intermodulation effect at a level comparable with that of the present atomic shot noise, we had to increase the noise of our quartz oscillator by two to three orders of magnitude. This work emphasizes the advantage of a continuous fountain in reducing intermodulation-related local oscillator noise and opens the possibility of reducing significantly the short-term instability via an increase in the atomic flux. To this end, we have designed and built a second continuous atomic fountain standard [12].

\section{ACKNOWLEDGMENTS}

METAS acknowledges the Centre National de la Recherche Scientifique (CNRS) and Laboratoire Kastler Brossel, unité mixte de recherche (UMR 8552) of the CNRS, for funding the stay of JG in its laboratory.

\section{REFERENCES}

[1] G.J. Dick, 'Local oscillator induced instabilities in trapped ion frequency standards", in Proceedings of the 19th Precise Time and Time Interval meeting (PTTI), Redendo Beach, CA, 1997, p. 133-147.

[2] G.J. Dick, J.D. Prestage, C.A. Greenhall and L. Maleki, "Local oscillator induced degradation of medium-term stability in passive atomic frequency standards", in Proceedings of the 22nd Precise Time and Time Interval meeting (PTTI), Vienna, VA, 1990, p. 487-508.

[3] C. Audoin, V. Candelier, and N. Dimarcq, "A limit to the frequency stability of passive frequency standards due to an intermodulation effect", IEEE Trans. Instrum. Meas., 40, 121-125 (1991).

[4] G. Santarelli, C. Audoin, A. Makdissi, Ph. Laurent, G.J. Dick, and A. Clairon, "Frequency stability degradation of an oscillator slaved to a periodically interrogated atomic resonator", IEEE Trans. Ultrason. Ferroelectr. Freq. Control, 45, 887-894 (1998).

[5] G. Santarelli, P. Lemonde, Ph. Laurent, and A. Clairon, A. G. Mann, C. Sheng, and A.N. Luiten, C. Salomon, "Quantum projection noise in an atomic fountain: a high stability cesium frequency standard", Phys. Rev. Lett. 82, 4619 (1999).

[6] A.G. Mann, C. Sheng, and A.N. Luiten, "Cryogenic sapphire oscillator with exceptionally high frequency stability", IEEE Trans. Instrum. Meas., 50, 519-521 (2001).

[7] S. Ohshima, T. Kurosu, T. Ikegami, and Y. Nakadan, "Multipulse operation of cesium atomic fountain", in Proceedings of the 5th Symposium on Frequency Standards and Metrology, Woods Hole, Massachussets, World Scientific Singapore (1995), p. 60-65.

[8] R. Legere and K. Gibble, "Quantum scattering in a Juggling Atomic Fountain", Phys. Rev. Lett. 81, 5780 (1998).

[9] A. Joyet, G. Mileti, G. Dudle, and P. Thomann, "Theoretical study of the Dick effect in a continuously operated Ramsey resonator", IEEE Trans. Instrum. Meas., 50, 150-155 (2001).

[10] A. Joyet, Aspects métrologiques d'une fontaine continue à atomes froids, Ph.D. thesis, University of Neuchâtel, 2003,

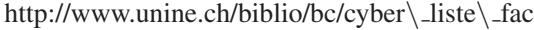
$\backslash$ _inst $\backslash$ FS $\backslash$ _physique.html

[11] Laurent-Guy Bernier, "New Real Time UTC(CH) Generation Scheme at METAS: Recent Progress in Control and Calibration Methods", these Proceedings.

[12] Ferenc Füzesi, Mark D. Plimmer, Gregor Dudle, Jocelyne Guéna and Pierre Thomann, "Design details of FOCS-2, an improved continuous cesium fountain frequency standard", these Proceedings. 